\title{
Fast and Effective Estimation of Narrowband Components for Bioelectrical Signals
}

\author{
Antonio Fasano ${ }^{1}$ and Valeria Villani ${ }^{2}$
}

\begin{abstract}
In this paper we propose a novel approach for estimating narrowband components from bioelectrical signals. The approach is based on the notion of modulated quadratic variation, introduced as a measure of variability for narrowband signals. The algorithm is the closed-form solution to a constrained convex optimization problem, where narrowband components are estimated tracking the slow variations around a central frequency in the measured signal. The approach is general and can be applied to any bioelectrical signal, either for diagnostic or denoising purposes. In this paper we assess its performance on ECG and EMG signals. Numerical results show its effectiveness in removing narrowband artifacts, such as power-line interference, while preserving signal morphology. It greatly outperforms conventional notch filtering. Moreover, it is also very fast, as its computational complexity is linear in the size of the vector to process.
\end{abstract}

\section{INTRODUCTION}

Bioelectrical signals are highly susceptible to electromagnetic interferences and can be contaminated by several kinds of noise and artifacts. In particular, power-line interference is ubiquitous in clinical environment. It is caused by supply plugs and cables, and sometimes can mask the real signal, especially those portions having low amplitude, such as late or evoked potentials in the case of electrocardiograms (ECGs) or electroencephalograms (EEGs), respectively [1]. Although various precautions can be taken (such as selection of a recording location with few surrounding electrical devices or appropriate shielding and grounding) and modern biomedical amplifiers have a very high common mode rejection ratio, recordings are often contaminated by residual power-line interference [1]-[5]. Thus, power-line interference rejection is an unavoidable step in any preprocessing of bioelectrical signals before clinical interpretation.

Quite a few solutions have been proposed in the literature addressing this problem. Most of them have been devised for ECG signals and, then, applied to suppress power-line noise from other bioelectrical signals [1], [3]. The standard approach involves the use of linear time-invariant notch filters [1], [5]-[7]. However, power-line amplitude and phase are time-varying. This makes classical approaches based on linear filtering ineffective, since they are unable to track slow variations around the fundamental $50 / 60 \mathrm{~Hz}$ and its harmonics. This causes a spread of its spectrum around $50 / 60 \mathrm{~Hz}$. Moreover, power-line interference is an in-band noise for any bioelectrical signal. Thus, on the one hand, the stopband

\footnotetext{
${ }^{1}$ Antonio Fasano is with the Faculty of Engineering, Università Campus Bio-Medico di Roma, Roma, Italy a.fasanoduni campus. it

${ }^{2}$ Valeria Villani is with the Dipartimento di Scienze e Metodi dell'Ingegneria, Università degli Studi di Modena e Reggio Emilia, Reggio Emilia, Italy valeria.villanieunimore.it
}

of notch filters should be as narrow as possible in order to minimize distortions in the filtered signal; on the other hand, a narrow stopband leads to ineffective filtering of power-line interference. To tackle this problem, adaptive filtering [8] [13] and other techniques [2] have been proposed.

In this paper we propose a novel approach to the estimation of narrowband components of bioelectrical signals, which is based on the notion of modulated quadratic variation. This is a suitable measure of variability for narrowband signals around a given frequency. The algorithm is the closed-form solution to a constrained convex optimization problem that exploits the modulated quadratic variation to track the slowly varying components around the central frequency. The approach is general and can be applied to estimate narrowband components of any bioelectrical signal, either for denoising or diagnostic purposes. Numerical results on ECG and electromyographic (EMG) signals highlight the effectiveness of the approach. Finally, the algorithm is also very fast, as its computational complexity is linear in the size of the vector to process. This makes it suitable for real-time applications as well for applications on devices with reduced computational complexity, such as handheld devices. Moreover, it allows processing of long recordings from Holter monitors.

\section{RATIONALE AND METHOD}

We consider sampled signals, so we will refer to the frequency $f$ considering the corresponding normalized frequency $\hat{f}=f / F_{s}$, where $F_{s}$ is the sampling rate. Narrowband components around a generic frequency $\hat{f}$ are characterized by the fact that their "variability" relative to the harmonic at $\hat{f}$ is lower than the corresponding "variability" of the measured bioelectric signal. Thus, provided that we introduce a suitable measure of "variability" around $\hat{f}$, narrowband components can be estimated searching for the signal closest, in some sense, to the measured signal, but exhibiting reduced variability. In this regard, we introduce the modulated quadratic variation (MQV) to measure the variability of a generic (complex) vector around a (normalized) central frequency $\hat{f}$.

Definition 1: Given a vector $\boldsymbol{z}=\left[z_{1} \cdots z_{n}\right]^{T} \in \mathbb{C}^{n}$, the modulated quadratic variation of $z$ around $\hat{f} \in(-0.5,0.5)$ is defined as

$$
[\boldsymbol{z}]_{\hat{f}} \doteq \sum_{k=1}^{n-1}\left|z_{k}-z_{k+1} e^{-j 2 \pi \hat{f}}\right|^{2}
$$

and is denoted by $[\boldsymbol{z}]_{\hat{f}}$. 
It is worth noting that the MQV generalizes the concept of quadratic variation, which is defined in [14] as a measure of variability for real vectors, and is used to effectively extract slowly varying components from bioelectrical signals (e.g., baseline wander from ECG recordings). Indeed, when $z$ is real and $\hat{f}=0$, (1) reduces to the quadratic variation. It is possible to prove that the definition (1) is well posed and the $\mathrm{MQV}$ is a consistent measure of variability for narrowband signals around the central frequency $\hat{f}$.

Introducing the $(n-1) \times n$ matrix $\mathcal{F}$ with entries

$$
\mathcal{F}_{h k}= \begin{cases}1 & k=h \\ -e^{-j 2 \pi \hat{f}} & k=h+1 \\ 0 & \text { otherwise }\end{cases}
$$

the MQV of $z$ can be equivalently expressed as

$$
[\boldsymbol{z}]_{\hat{f}}=\boldsymbol{z}^{H} \mathcal{F}^{H} \mathcal{F} \boldsymbol{z}=\|\mathcal{F} \boldsymbol{z}\|^{2}
$$

where $(\cdot)^{H}$ denotes the conjugate transpose and $\|\cdot\|$ is the Euclidean norm for complex vectors.

Let $\boldsymbol{q}$ be the vector collecting $n$ samples of a measured signal and denote by $z$ the vector of estimated narrowband components around $\hat{f}$. These components can be estimated searching for the signal components that, with respect to the measured signal $\boldsymbol{q}$, have reduced variability relative to $\hat{f}$. This amounts to searching for the components that are "close" to the observed signal, but have reduced MQV around $\hat{f}$. Measuring closeness in terms of the $l^{2}$ norm, we propose to estimate the narrowband components of $\boldsymbol{q}$ around $\hat{f}$ by solving the following convex optimization problem

$$
\left\{\begin{array}{cl}
\underset{\boldsymbol{z} \in \mathbb{C}^{\mathbb{z}}}{\operatorname{minimize}} & \|\boldsymbol{z}-\boldsymbol{q}\|^{2} \\
\text { subject to } & {[\boldsymbol{z}]_{\hat{f}} \leq \rho}
\end{array}\right.
$$

where $\rho$ is a nonnegative constant that controls the MQV of the estimated components. Its value is chosen in accordance with the peculiarity of the problem and should satisfy $\rho<[\boldsymbol{q}]_{\hat{f}}$, to avoid trivial ${ }^{1}$ solutions. Note that we do not need to know in advance the appropriate value for $\rho$ in any particular problem. In fact, the solution of (4) can be expressed in terms of a parameter that is related to $\rho$. In this way, narrowband components can be estimated without caring about $\rho$, by parametrically reducing the MQV of the solution $z$ to the desired level.

The solution of (4) is given by

$$
\boldsymbol{z}=\left(\boldsymbol{I}+\lambda \mathcal{F}^{H} \mathcal{F}\right)^{-1} \boldsymbol{q}
$$

where $\boldsymbol{I}$ denotes the $n \times n$ identity matrix, and $\lambda$ is a nonnegative parameter in one-to-one correspondence with $\rho$. In (5) the matrix inverse exists for any $\lambda \geq 0$, since $\mathcal{F}^{H} \mathcal{F}$ is positive semidefinite. It is interesting that the solution to (4) is a linear operator acting on $\boldsymbol{q}$. The parameter $\lambda$ controls the MQV of the solution $z$, i.e., the degree of variability of the estimated narrowband components. Note that $\boldsymbol{z} \in \mathbb{C}^{n}$ even though $\boldsymbol{q} \in \mathbb{R}^{n}$, as a consequence of

${ }^{1}$ When $\rho \geq[\boldsymbol{q}]$ the solution $\boldsymbol{z}=\boldsymbol{q}$ coincides with the measured signal.

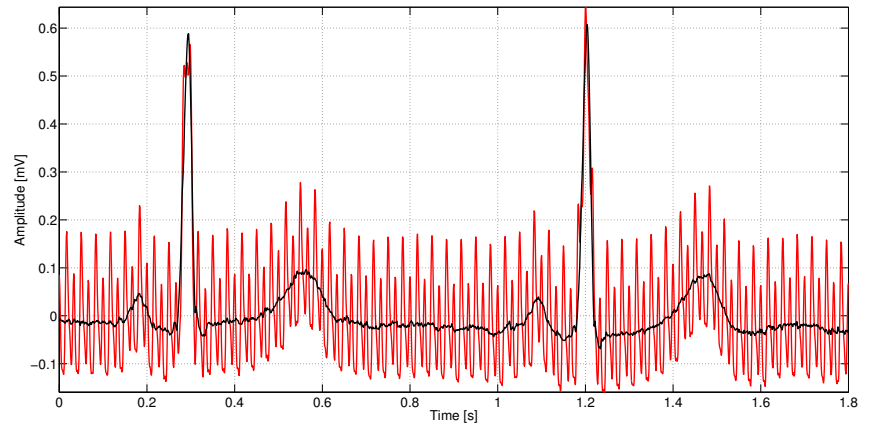

Fig. 1. Real reference ECG ( $\boldsymbol{q}_{0}$, black) corrupted with narrowband artifacts centered at $30 \mathrm{~Hz}, 60 \mathrm{~Hz}$, and $120 \mathrm{~Hz}$ (red), with $\mathrm{SIR}_{0}=0 \mathrm{~dB}$.

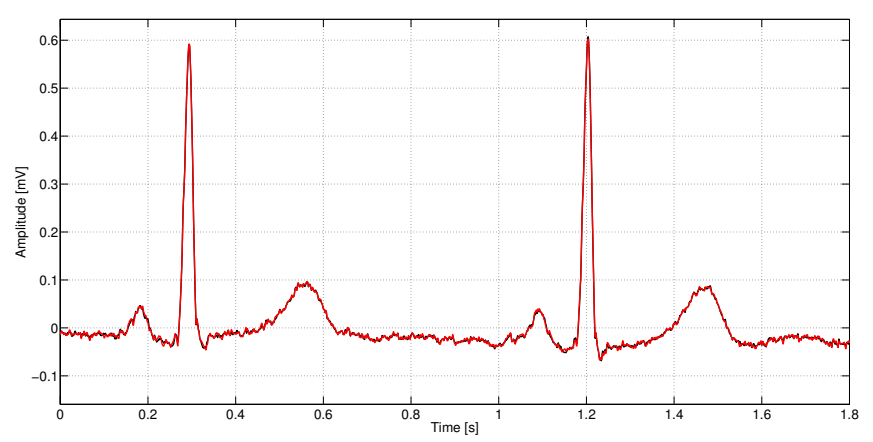

Fig. 2. Denoised ECG (red) superimposed on the reference ECG (black).

the fact that (5) returns the narrowband components around $\hat{f}$, where $\hat{f} \in(-0.5,0.5)$. Real signals have symmetric spectrum and narrowband components are both at $\hat{f}$ and $-\hat{f}$. Thus, considering both the contributions around $\hat{f}$ and $-\hat{f}$, it is possible to prove that to estimate the (real) narrowband components of $\boldsymbol{q} \in \mathbb{R}^{n}$ around $|\hat{f}|$, denoted by $\boldsymbol{q}_{\hat{f}} \in \mathbb{R}^{n}$, the following formula applies

$$
\boldsymbol{q}_{\hat{f}}=2 \operatorname{Re}\{\boldsymbol{z}\}
$$

where $z$ is given by (5).

Finally, some remarks on computational issues. Since $\boldsymbol{I}+\lambda \mathcal{F}^{H} \mathcal{F}$ is Hermitian, positive-definite, tridiagonal, (5) can be computed very efficiently with complexity $O(n)$ [15], i.e., linear in the size of the vector $\boldsymbol{q}$. Moreover, complexity does not depend on the nature of the signal to be processed. To give an idea of the performance, an efficient implementation of (5) in MATLAB (ver. 8.3), running over a PC equipped with a $2.6 \mathrm{GHz}$ Core i5 processor, takes about $0.62 \mathrm{~s}$ to process a record of $10^{7}$ double precision floating point samples. This property makes the proposed approach suitable for real-time applications as well as for applications on devices with reduced computing power, e.g., handheld devices.

\section{SIMULATION RESULTS}

The performance of the proposed approach has been investigated on real ECG and EMG signals corrupted by synthetic narrowband artifacts.

As regards ECG signals, we considered the ECG record s0306lrem from the PTB Diagnostic ECG Database [16] of 


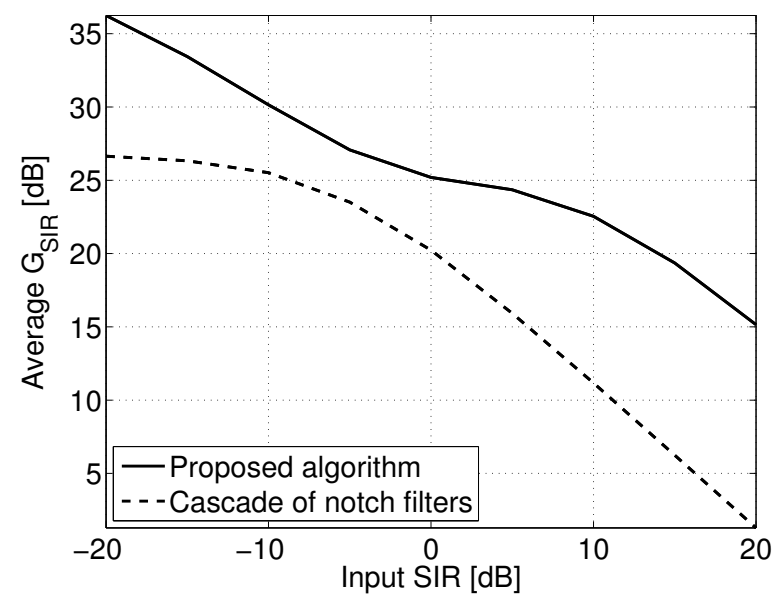

Fig. 3. Average SIR gain $G_{\text {SIR }}$ versus input SIR on ECG signals: comparison between the proposed approach and a cascade of notch filters.

PhysioNet [17]. It was sampled at $1 \mathrm{kHz}$ with 16-bit resolution and has been used as a reference in our simulations since it has negligible harmonic components at $60 \mathrm{~Hz}$ and multiples. This reference signal, denoted by $\boldsymbol{q}_{0}$, was corrupted by narrowband artifacts generated as sine waves having timevarying amplitude, centered at $30 \mathrm{~Hz}, 60 \mathrm{~Hz}$, and $120 \mathrm{~Hz}$ : they model a generic in-band artifact and the first and second harmonics of power-line interference, respectively. Timevarying amplitudes were rendered by low-pass filtering three independent realizations of white Gaussian noise with cut-off frequency $2 \mathrm{~Hz}$. Mean values and standard deviations of the three realizations are in the ratio $\mu_{60} / \mu_{30}=\sigma_{60} / \sigma_{30}=2$ and $\mu_{60} / \mu_{120}=\sigma_{60} / \sigma_{120}=3$, where the subscript indicates the central frequency of the artifacts. The resulting corrupted signal is denoted by $\boldsymbol{q}=\boldsymbol{q}_{0}+\boldsymbol{d}$, where $\boldsymbol{d}$ is the sum of the three harmonic components, and has a signal-to-interference ratio (SIR) $\operatorname{SIR}_{0}=10 \log \frac{\left\|\boldsymbol{q}_{0}\right\|^{2}}{\|\boldsymbol{d}\|^{2}}=0 \mathrm{~dB}$. A portion of the corrupted signal $\boldsymbol{q}$ is reported (red) in Fig. 1 together with the reference record $\boldsymbol{q}_{0}$ (black).

In Fig. 2 we show the ECG signal (red) after artifact rejection using the proposed approach, denoted by $\boldsymbol{x}$, superimposed on the reference ECG $\boldsymbol{q}_{0}$ (black). The parameter $\lambda$ was roughly set to $10^{8}$ without any optimization. As the figure highlights, the proposed approach effectively suppresses narrowband artifacts, without introducing noticeable distortion in the ECG morphology.

Performance is measured in terms of SIR gain

$$
\mathrm{G}_{\mathrm{SIR}}=\mathrm{SIR}_{\mathrm{s}}-\mathrm{SIR}_{0}=10 \log \frac{\|\boldsymbol{d}\|^{2}}{\left\|\boldsymbol{x}-\boldsymbol{q}_{0}\right\|^{2}}
$$

where $\operatorname{SIR}_{\mathrm{s}}=10 \log \frac{\left\|\boldsymbol{q}_{0}\right\|^{2}}{\left\|\boldsymbol{x}-\boldsymbol{q}_{0}\right\|^{2}}$ is the signal-to-interference ratio after artifact suppression. By $\left(\boldsymbol{x}-\boldsymbol{q}_{0}\right)$ we regard as noise affecting $\boldsymbol{x}$ both the residual interference and the reconstruction error. Regarding the signals in Fig. 2, the proposed approach achieves an improvement of about $26 \mathrm{~dB}$, over an initial SIR as low as $0 \mathrm{~dB}$. In the same setting, a cascade of three IIR notch filters with attenuations $26 \mathrm{~dB}$,

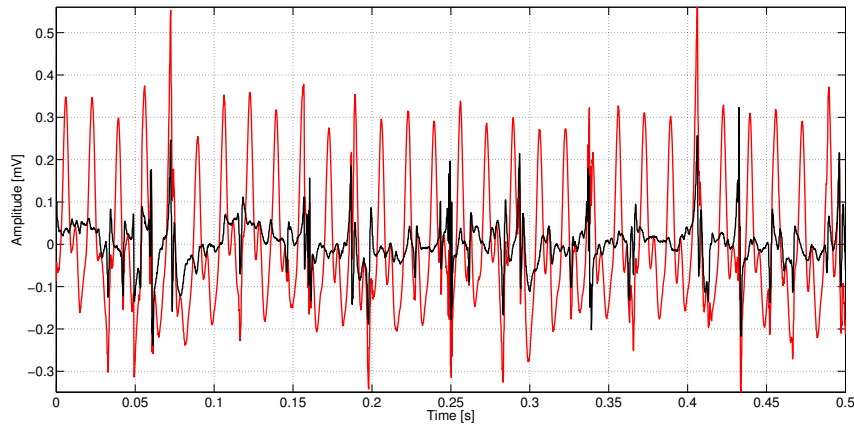

Fig. 4. Real reference EMG ( $\boldsymbol{q}_{0}$, black) corrupted with narrowband artifacts centered at $60 \mathrm{~Hz}, 120 \mathrm{~Hz}$, and $180 \mathrm{~Hz}$ (red), with $\mathrm{SIR}_{0}=-5 \mathrm{~dB}$.

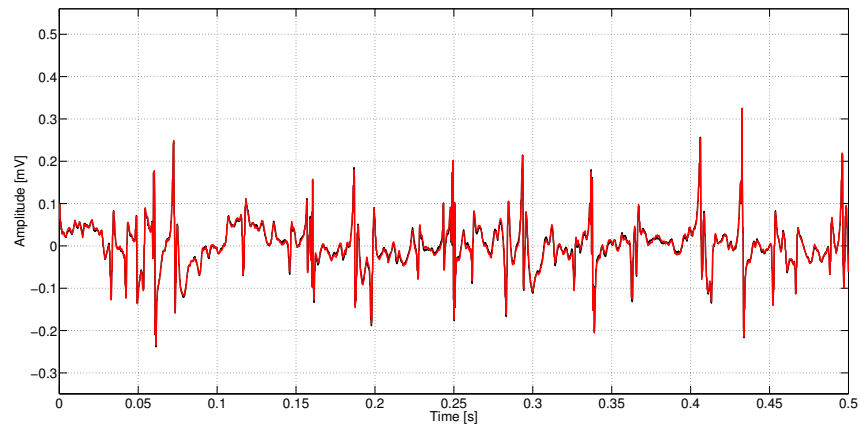

Fig. 5. Denoised EMG (red) superimposed on the reference EMG (black).

$54 \mathrm{~dB}$ and $54 \mathrm{~dB}$, respectively, at the central frequencies of the interference achieves an improvement of only $20 \mathrm{~dB}$.

In order to evaluate how gain varies as input SIR changes, in Fig. 3 we report the average SIR gain versus input SIR, when the reference ECG $\boldsymbol{q}_{0}$ is corrupted by narrowband artifacts with SIR ranging from $-20 \mathrm{~dB}$ to $20 \mathrm{~dB}$. For each input SIR we averaged the gain $\mathrm{G}_{\mathrm{SIR}}$ over 30 realizations of artifact. The parameter $\lambda$ was set as the one that entails the minimum of (6) for each noise realization. This choice is motivated by the need to determine the limit performance of the proposed algorithm. However, it can be shown that the choice of $\lambda$ is not a critical issue. As Fig. 3 highlights, the proposed algorithm is very effective in suppressing narrowband artifacts in ECG signals and achieves considerable gain over the whole range of input SIR. It is worth noting that the approach based on MQV reduction is able to reject both out-of-band and in-band noise. In this regard, notch filtering cannot reject in-band noise without altering the signal. In particular, it introduces ringing artifacts at the offset of QRS complexes [1]. For comparison, we report in Fig. 3 the average SIR gain achieved by the cascade of notch filters considered above. As the figure highlights, the proposed algorithm is very effective in suppressing narrowband artifacts and greatly outperforms notch filtering for all values of input SIR.

As regards EMG signals, we considered an EMG recorded from a 44 years old man without history of neuromuscular disease [17]. The record was sampled at $4 \mathrm{kHz}$ and highpass filtered with cut-off frequency $20 \mathrm{~Hz}$. It does not present noticeable power-line interference, and we used it as a reference, denoted by $\boldsymbol{q}_{0}$. It was corrupted by narrowband 


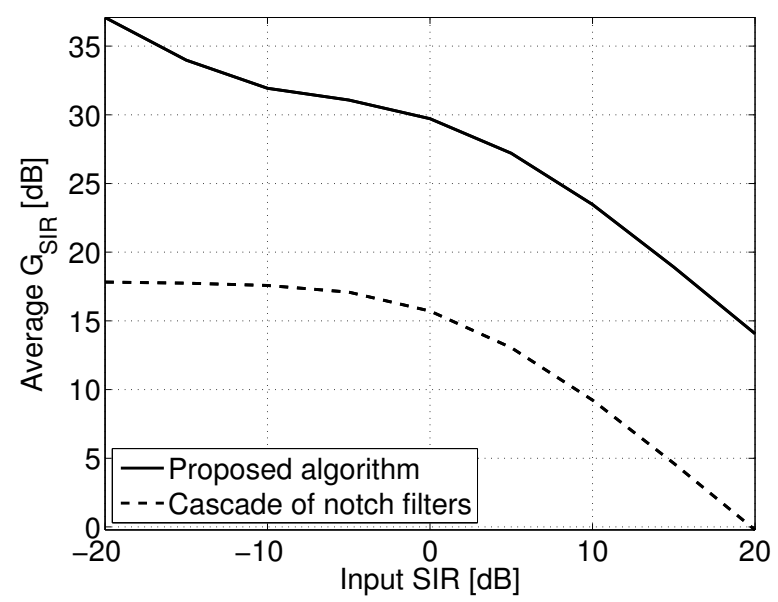

Fig. 6. Average SIR gain $\mathrm{G}_{\mathrm{SIR}}$ versus input SIR on EMG signals: comparison between the proposed approach and a cascade of notch filters.

artifacts generated as sine waves having time-varying amplitude, centered at $60 \mathrm{~Hz}, 120 \mathrm{~Hz}$ and $180 \mathrm{~Hz}$, modeling the first three harmonics of power-line noise. Time-varying amplitudes were generated as described above in the case of ECG. Mean values and standard deviations of the three realizations of noise are in the ratio $\mu_{60} / \mu_{120}=\sigma_{60} / \sigma_{120}=$ 2 and $\mu_{60} / \mu_{180}=\sigma_{60} / \sigma_{180}=3$. In Fig. 4 we show a portion of the reference $\boldsymbol{q}_{0}$ (black) and the corresponding corrupted record (red). It is heavily affected by artifacts and exhibits $\mathrm{SIR}_{0}=-5 \mathrm{~dB}$. The result of artifacts rejection by means of MQV reduction (red) is reported in Fig. 5, together with the reference EMG. The parameter $\lambda$ was roughly set to $10^{8}$, as in the case of ECG. The gain $\mathrm{G}_{\mathrm{SIR}}$ achieved by the proposed approach is $32 \mathrm{~dB}$ and, as Fig. 5 shows, the original waveform is restored without noticeable distortion. In the same setting, a cascade of three IIR notch filters with attenuations $15 \mathrm{~dB}, 15 \mathrm{~dB}$ and $23 \mathrm{~dB}$ at the central frequencies of the interference returns a gain $\mathrm{G}_{\mathrm{SIR}}=17 \mathrm{~dB}$, which is $15 \mathrm{~dB}$ less than the proposed approach.

Finally, we computed the average SIR gain versus input SIR, when the reference EMG is corrupted by narrowband artifacts with SIR ranging from $-20 \mathrm{~dB}$ to $20 \mathrm{~dB}$. Results are shown in Fig. 6 in comparison with the cascade of notch filters considered above. Gains are averaged over 30 realizations of artifact. The figure confirms the effectiveness of the approach, which achieves remarkable gain in comparison with the cascade of notch filters, over the whole range of input SIR. The improvement exceeds $14 \mathrm{~dB}$. Moreover, considering separately all realizations of artifact (30 realizations for each of the 9 input SIRs, for a total of 270 independent realizations), the minimum improvement of the proposed algorithm over notch filtering exceeds $12 \mathrm{~dB}$ in all cases, which is notable.

\section{CONCLUSIONS}

In this paper we propose a novel approach for estimating narrowband components from bioelectrical signals. The approach is based on the notion of modulated quadratic variation, which is introduced as a consistent measure of variability for narrowband signals. The algorithm is the closed-form solution to a constrained convex optimization problem that exploits the modulated quadratic variation to track the slowly varying components around a given central frequency. The approach is general and can be applied to any bioelectrical signal, either for denoising or diagnostic purposes. Here we assess the performance on ECG and EMG signals. Results confirm the effectiveness of the approach and highlight its ability in removing narrowband artifacts while preserving signal morphology. Moreover, it is very fast, even on long recordings, thus being well suited for real-time applications and implementation on devices with reduced computational power, such as handheld devices.

\section{REFERENCES}

[1] L. Sörnmo and P. Laguna, Bioelectrical Signal Processing in Cardiac and Neurological Applications. Elsevier Academic Press, 2005.

[2] C. Levkov, G. Mihov, R. Ivanov, I. Daskalov, I. Christov, and I. Dotsinsky, "Removal of power-line interference from the ECG: a review of the subtraction procedure," BioMed. Eng. OnLine, vol. 4, no. 50, 2005.

[3] R. Merletti and P. Parker, Eds., Electromyography: Physiology, Engineering, and Non-Invasive Applications. Wiley-IEEE Press, 2004.

[4] G. D. Clifford, F. Azuaje, and P. McSharry, Eds., Advanced Methods and Tools for ECG Data Analysis. Artech House, Inc., 2006.

[5] R. E. Gregg, S. H. Zhou, J. M. Lindauer, E. D. Helfenbein, and K. K. Giuliano, "What is inside the electrocardiograph?" J. Electrocardiol., vol. 41, pp. 8-14, 2008.

[6] S. C. Pei and C. C. Tseng, "Elimination of AC interference in electrocardiogram using IIR notch filter with transient suppression," IEEE Trans. Biomed. Eng., vol. 42, no. 11, pp. 1128-1132, 1995.

[7] J. A. Van Alsté and T. S. Schilder, "Removal of base-line wander and power-line interference from the ECG by an efficient FIR filter with a reduced number of taps," IEEE Trans. Biomed. Eng., vol. 32, no. 12, pp. 1052-1060, 1985.

[8] B. Widrow, J. R. Glover, J. M. McCool, J. Kaunitz, C. Williams, R. H. Hean, J. R. Zeidler, E. J. Dong, and R. C. Goodlin, "Adaptive noise cancelling: principles and applications," Proc. IEEE, vol. 63, no. 12, pp. 1692-1716, 1975.

[9] N. V. Thakor and Y. S. Zhu, "Applications of adaptive filtering to ECG analysis: Noise cancellation and arrhythmia detection," IEEE Trans. Biomed. Eng., vol. 38, no. 8, pp. 785-794, 1991.

[10] P. S. Hamilton, "A comparison of adaptive and non-adaptive filters for reduction of power line interference in the ECG," IEEE Trans. Biomed. Eng., vol. 43, no. 1, pp. 105-109, 1996.

[11] A. K. Ziarani and A. Konrad, "A nonlinear adaptive method of elimination of power line interference in ECG signals," IEEE Trans. Biomed. Eng., vol. 49, no. 6, pp. 540-547, 2012.

[12] S. M. M. Martens, M. Mischi, S. G. Oei, and J. W. M. Bergmans, "An improved adaptive power line interference canceller for electrocardiography," IEEE Trans. Biomed. Eng., vol. 53, no. 11, pp. 2220-2231, 2006.

[13] M. H. Costa and M. C. Tavares, "Removing harmonic power line interference from biopotential signals in low cost acquisition systems," Comp. Biol. Med., vol. 39, pp. 519-526, 2009.

[14] A. Fasano and V. Villani, "Baseline wander removal for bioelectrical signals by quadratic variation reduction," Signal Process., vol. 99, no. 48-57, 2014

[15] G. H. Golub and C. F. van Loan, Matrix Computation, 3rd ed. The Johns Hopkins University Press, 1996.

[16] R. Bousseljot, D. Kreiseler, and A. Schnabel, "Nutzung der EKGsignaldatenbank CARDIODAT der PTB über das internet," Biomedizinische Technik, vol. 40, no. Suppl. 1, p. S 317, 1995.

[17] A. L. Goldberger, L. A. N. Amaral, L. Glass, J. M. Hausdorff, P. C. Ivanov, R. G. Mark, J. E. Mietus, G. B. Moody, C. K. Peng, and H. E. Stanley, "PhysioBank, PhysioToolkit, and PhysioNet: Components of a new research resource for complex physiologic signals," Circulation, vol. 101, no. 23, pp. e215-e220, 2000. 